\title{
Caracterización de los ecotopos cafeteros colombianos en el
} Triángulo del Café

\section{CHARACTERIZATION OF COLOMBIAN COFFEE ECOTOPES IN THE COFFEE TRIANGLE REGION}

\author{
CARACTERIZAÇÃO DOS ECÓTOPOS CAFEICULTORES COLOMBIANOS NO \\ TRIANGULO DO CAFÉ
}

Olga Lucía Ocampo López ${ }^{2}$ Karen Castañeda Peláez ${ }^{3}$ Jorge Julián Vélez Upegui ${ }^{4}$
Para citar este artículo: Ocampo López, O. L., Castañeda Peláez, K. y Vélez Upegui, J. J. (2017). Caracterización de los ecotopos cafeteros colombianos en el Triángulo del Café. Perspectiva Geográfica, 22(1), 89-108. doi: 10.19053/01233769.6100.
Recepción:

9 de septiembre de 2016

Evaluación:

5 de diciembre de 2016

\section{Aprobación:}

26 de diciembre de 2016

\section{Resumen}

El Triángulo del Café, en Colombia, está conformado por los departamentos de Caldas, Quindío y Risaralda. Su diversidad geográfica, climática y de suelos permite identificar varios ecotopos cafeteros que se determinan por respuestas similares en cuanto a productividad. La caracterización de los ecotopos que se presenta en este artículo fue posible por la disponibilidad de información geográfica de libre acceso, producto de la Ley de Transparencia y del Derecho de Acceso a la Información Pública Nacional (Ley 1712 de 2014).

1 Este artículo es resultado de la investigación "Calibración y validación de modelos de cultivo del café para la evaluación de los efectos de variabilidad climática", financiado por la Universidad Autónoma de Manizales y Colciencias, que hace parte del proyecto de investigación "Modelación hidrológica y Agronómica de los impactos del cambio y la variabilidad climática en la productividad cafetera de Caldas", financiado por la Universidad Autónoma de Manizales y la Universidad Nacional de Colombia, sede Manizales.

2 Candidato a doctor en Ingeniería con maestría en Ingeniería de la Universidad Nacional de Colombia, sede Manizales. Docente asociado de la Universidad Autónoma de Manizales e investigador asociado de los grupos en Diseño Mecánico y Desarrollo Industrial y Desarrollo Regional Sostenible. olocampo@autonoma.edu.co

3 Bióloga, joven investigadora de la Universidad Autónoma de Manizales, Grupo de Investigación Diseño Mecánico y Desarrollo Industrial. kpelaez@autonoma.edu.co

4 Doctor en Planificación y Gestión de Recursos Hidráulicos de la Universidad Politécnica de Valencia. Profesor asociado de la Universidad Nacional de Colombia sede Manizales. Investigador senior del Grupo de Trabajo Académico en Ingeniería Hidráulica y Ambiental. jjvelezu@unal.edu.co 
La información utilizada para la caracterización está relacionada con geografia, hidrología, clima, geología, suelos y biomas. El análisis fue realizado empleando las herramientas de los sistemas de información geográfica. Los resultados permiten una mejor comprensión de los factores climáticos y geográficos que son determinantes de la productividad cafetera y, a su vez, brindan lineamientos para la ordenación y planificación del territorio.

Palabras clave: café, ecotopos cafeteros, factores climáticos, factores geográficos.

\section{Abstract}

The Colombian coffee triangle comprises the departments of Caldas, Quindío and Risaralda. Geographical, climatic and soil diversity of the region allows to identify several coffee ecotopes that are determined by similar responses in terms of productivity. The characterization of the ecotopes shown in this paper was possible due to the availability of freely accessible geographic information, according to the law on transparency and the right to public information (Law 1712 of 2014). The information used for the characterization is related to geography, hydrology, climate, geology, soils and biomes. Geographic information systems tools were used for this analysis. Results allow a better understanding of climatic and geographical factors that are determinants for coffee productivity. Thus, guidelines for management and land use planning are provided.

Keywords: coffee, ecotopes, climatic factors, geographic factors.

\section{Resumo}

O Triangulo do Café, na Colômbia, é formado pelos Estados de Caldas, Quindio e Risaralda. Sua diversidade geográfica, climática e do solo permite identificar vários ecótopos do café que se determinam por respostas similares quanto a produtividade. A caracterização dos ecótopos que se apresentam neste artigo foi possível pela disponibilidade de informação geográfica de livre acesso, produto da Lei de Transparência e Direito de Acesso a Informação Pública Nacional (Lei 1712 de 2014). A informação utilizada para a caracterização está relacionada com geografia, hidrologia, clima, geologia, solo e biomas. A análise foi realizada utilizando as ferramentas dos sistemas de informação geográfica. Os resultados permitem uma melhor compreensão dos fatores climáticos e geográficos que são determinantes para produtividade do café e por sua vez fornecer lineamento para ordenação e planificação do território.

Palavra-chave: café, ecótopos, cafeicultores, fatores climáticos e fatores geográficos. 


\section{Introducción}

Las estadísticas de la caficultura colombiana revelan la importancia del café para el desarrollo nacional: 560.000 caficultores cultivan café en 590 municipios y emplean de manera directa a 530.000 personas, mientras que 2.500.000 personas dependen del cultivo (Federación Nacional de Cafeteros, 2015). En Colombia, las plantaciones se encuentran distribuidas en las vertientes de los tres ramales de la cordillera de los Andes, desde el Ecuador geográfico hasta los $12^{\circ}$ de latitud norte y entre los $72^{\circ}$ y $78^{\circ}$ de longitud oeste. La franja altitudinal comprende desde 1.000 a 2.000 m. s. n. m., dentro de las cuales se tiene una zona óptima entre 1.300 y 1.700 m. s. n. m., una zona marginal baja de $1.000-1.300$ m. s. n. m. y una zona marginal alta entre 1.700 a 2.000 m. s. n. m. (Jaramillo, 2005; Calambas, 2009).

El Paisaje Cultural Cafetero ha sido declarado Patrimonio Cultural de la Humanidad por la Unesco debido a sus rasgos distintivos, a saber: carácter familiar, intensidad de mano de obra, calidad del producto, impacto en la economía regional, capital social e institucionalidad (Ministerio de Cultura, 2011). Los municipios cafeteros de los departamentos de Caldas, Quindío y Risaralda, que conforman el denominado Triángulo del Café, hacen parte de la declaración de Paisaje Cultural Cafetero, lo que exalta la importancia de un mayor conocimiento de las zonas cafeteras.

Estas zonas, a su vez, se encuentran clasificadas por ecotopos cafeteros. Según Gómez, Caballero y Baldión (1991), un ecotopo es "una región agroecológica, delimitada geográficamente, teniendo en cuenta condiciones predominantes de clima, suelo y relieve, donde se obtiene una respuesta biológica similar del cultivo del café" (p. 7). Las variadas condiciones edáficas y climáticas de estos ecotopos se reflejan en el desarrollo de la caficultura y en la producción (Gómez, Caballero y Baldión, 1991; Arcila, Farfán, Moreno, Salazar e Hincapié, 2007). En consecuencia, es necesario comprender la variabilidad espacial de los factores que pueden influir en la producción cafetera (Arcila et al., 2007), de manera que se tomen en consideración para la planeación de estrategias de producción y proporcionen herramientas para la toma de decisiones en el cultivo.

La caracterización de los ecotopos cafeteros desarrollada por Gómez Caballero y Baldión (1991) presenta una descripción de cada ecotopo y sus principales características climáticas, edáficas, productivas y geológicas. Teniendo en cuenta la información pública disponible en el país, es posible actualizar esta línea base de manera que brinde insumos para mejorar la delimitación de los ecotopos cafeteros y la planificación del territorio. Como plantea Salinas Chaves (2013), esta es una vía para alcanzar el desarrollo sostenible; por su complejidad, se requiere un enfoque holístico, sistémico y estratégico que permita tomar decisiones adecuadas para promover el desarrollo de forma integral. En territorios agrícolas y rurales, los sistemas de información geográfica (SIG) y la cartografía facilitan la comprensión de esta complejidad territorial y, por tanto, pueden ser empleados para su caracterización.

Este artículo es producto de una investigación que tuvo por objeto caracterizar los ecotopos cafeteros considerando el análisis espacial de factores geográficos y climáticos que inciden en la producción cafetera. Se tomaron, como caso de estudio, los departamentos de Caldas, Quindío y Risaralda, que conforman el Triángulo del Café. Inicialmente, se presenta una revisión de estos factores; luego, se describe la metodología y los resultados obtenidos mediante el procesamiento, a través de sistemas de información geográfica, de la información secundaria disponible en las plataformas del Sistema de Información Ambiental de Colombia (SIAC, 2016) y el Sistema de Información Geográfica para la Planeación y el Ordenamiento Territorial (SIG-OT), cuyo acceso fue posible por la Ley 1712 del 6 de marzo del 2014 sobre la transparencia y el derecho a la información pública nacional. Esta ley ha llevado a un cambio estructural en el acceso a la información geográfica; en consecuencia, permite avanzar en la comprensión del territorio, conocer sus potenciales y limitaciones y sus posibles aplicaciones, como se presenta en este estudio 
de caracterización de los ecotopos cafeteros en el Triángulo del Café.

\section{Factores climáticos y geográficos que indicen en la productividad cafetera}

La productividad del cafetal está determinada por la variedad, la interacción con el medio ambiente y las prácticas de manejo (Arcila et al., 2007). Cada región cafetera tiene un clima que conduce a un comportamiento específico de la planta y determina su productividad (Jaramillo, 2005). Entre los factores climáticos y geográficos que influyen en el cultivo del café se encuentran la precipitación, la temperatura, la humedad relativa, la radiación y el brillo solar, la velocidad y la dirección del viento, la altitud y la latitud (Havlin, Beaton, Tisdale y Nelson, 1999; Arcila et al., 2007). Estas variables determinan la disponibilidad de agua y energía, que juegan un papel relevante en la producción y son necesarias para el crecimiento y el desarrollo de la planta (Ritchi, 1991).

Aunque el café es una planta altamente adaptada a los patrones climáticos de los trópicos, es sensible a los cambios en las condiciones climáticas (Carr, 2001; Turbay, Nantes, Jaramillo, Vélez y Ocampo, 2014) porque requiere un microclima específico para su desarrollo (Gliessman, 2002). Es así como los cambios en la temperatura del aire afectan la floración, que también está influenciada por el fotoperiodo y la distribución de los períodos húmedos (Camayo-Vélez, Chávez-Córdoba, Arcila-Pulgarín, y Jaramillo-Robledo, 2003; Crisosto, Grantz y Meinzer, 1992; Paes, 1985; Ramírez et al., 2011). Si bien el café requiere un período definido de sequía, un estrés hídrico extendido puede dañar la planta (Lin, 2007; Ocampo, 2017).

Diferentes autores (Arcila et al., 2007; Calambas, 2009; Jaramillo, 2005; Poveda et al, 2014; Ocampo, 2017) presentan una revisión de los principales factores que afectan el potencial de producción: los factores climáticos óptimos que influyen en el cultivo del café son las lluvias entre 1.800 y 2.800 $\mathrm{mm}$ por año, las temperaturas entre 18 a $23^{\circ} \mathrm{C}$, el brillo solar, mayor a seis horas de luz por día y cerca de las 2.000 horas de luz por año, ocho a trece horas de iluminación necesarias para la floración y una humedad relativa del 70 al $85 \%$ (Ocampo, 2017).

\section{Metodología}

La unidad de análisis fue el Triángulo del Café, cuya localización se representa en la Figura 1; comprende los departamentos de Caldas, Quindío y Risaralda, que están conformados por 27, 12 y 14 municipios respectivamente. Para este estudio, se tomó como punto de partida la identificación de los ecotopos cafeteros realizada por el Centro Nacional de Investigaciones del Café (Cenicafé, 2015) en la zona objeto de análisis, y se representan en la Figura 1 (d). Las variables que se consideraron para la caracterización y su método de estimación se resumen en la Tabla 1. 

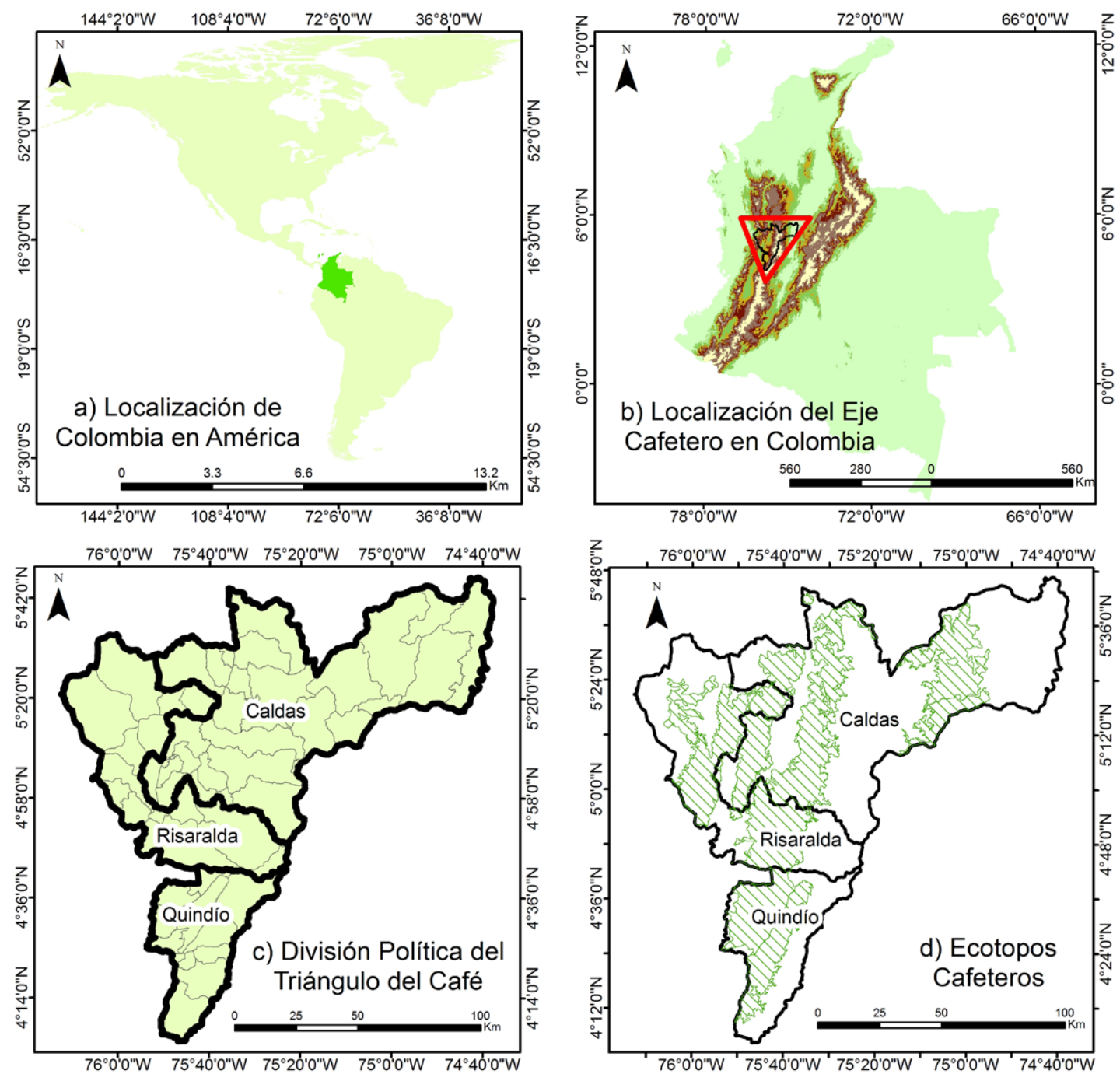

Figura 1. Localización de los ecotopos cafeteros en el Triángulo del Café.

Fuente: Elaboración propia a partir de Cenicafé (2015), National Aeronautics and Space Administration (NASA, 2015) y SIAC (2016). 
TABLA 1. VARIABLES EMPLEADAS EN LA CARACTERIZACIÓN

\begin{tabular}{llll} 
Variables & Unidades & Método de estimación & Fuente \\
\hline Altitud & m s. n. m. & Modelo de elevación digital del terreno & NASA (2015) \\
\hline Biomas y agroecosistemas & $\%$ área & $\begin{array}{l}\text { Mapa de ecosistemas generales de Colom- } \\
\text { bia, } 1998\end{array}$ & SIAC (2016) \\
\hline
\end{tabular}

Parques nacionales naturales

Reservas forestales protectoras nacionales

Parque natural regional

Áreas de reserva y protección Shape de áreas

Reservas forestales protectoras regionales

SIAC (2016)

Distrito regional de manejo integrado

Distrito de conservación de suelos Reservas naturales de la sociedad civil

\begin{tabular}{|c|c|c|c|}
\hline Coberturas & \% área & Mapa de cobertura de la tierra, 2008 & SIG-OT (2016) \\
\hline Geología & & Mapa geológico colombiano & $\begin{array}{l}\text { Servicio Geológico Co- } \\
\text { lombiano (2016) }\end{array}$ \\
\hline Vocación de suelos & Shape de áreas & Mapa de vocación de uso de suelo & $\begin{array}{l}\text { Unidad de Planificación } \\
\text { Rural Agropecuaria } \\
\text { (2016) }\end{array}$ \\
\hline Conflictos de uso del suelo & Shape de áreas & Conflictos de uso del suelo & SIG-OT (2016) \\
\hline Erosión & Shape de áreas & $\begin{array}{l}\text { Zonificación de la degradación de suelos } \\
\text { por erosión. Área continental de Colombia. } \\
\text { Escala 1:100.000. Línea base 2010-2011 }\end{array}$ & SIAC (2016) \\
\hline Radiación solar & $\mathrm{kWh} / \mathrm{m}^{2}$ & $\begin{array}{l}\text { Radiación solar global, promedio mul- } \\
\text { tianual }\end{array}$ & SIAC (2016) \\
\hline Humedad relativa & $\%$ & Correlación con altitud & Ocampo y Vélez (2015) \\
\hline Precipitación & $\mathrm{mm}$ & $\begin{array}{l}\text { Precipitación total anual según el IDEAM, } \\
2012\end{array}$ & SIAC (2016) \\
\hline Temperaturas & ${ }^{\circ} \mathrm{C}$ & $\begin{array}{l}\text { Línea base: temperaturas mínimas y } \\
\text { máximas }\end{array}$ & WorldClim (2016) \\
\hline Clasificación climática & Shape de áreas & Mapa de clasificación climática de Lang & SIAC (2016) \\
\hline Cuencas hidrográficas & Shape de áreas & Demanda hídrica total, 2014 & SIAC (2016) \\
\hline Evapotranspiración & $\mathrm{mm} / \mathrm{d}$ & Modelo en función de la latitud & Jaramillo (2005) \\
\hline Escorrentía & $\mathrm{mm}$ & $\begin{array}{l}\text { Escorrentía mensual de Colombia, Escena- } \\
\text { rio Año medio, Estudio Nacional del Agua } \\
\text { (ENA, 2010) }\end{array}$ & SIAC (2016) \\
\hline $\begin{array}{l}\text { Índice de alteración potencial de } \\
\text { la calidad }\end{array}$ & Shape de áreas & Índice de alteración potencial de la calidad & SIAC (2016) \\
\hline Índice de uso de agua & Shape de áreas & $\begin{array}{l}\text { Índice de uso del agua condiciones hídricas } \\
\text { por subzonas hidrográficas, } 2010\end{array}$ & SIAC (2016) \\
\hline Índice de vulnerabilidad hídrica & Shape de áreas & $\begin{array}{l}\text { Índice de vulnerabilidad hídrica, } \\
\text { condiciones año seco, } 2010 \\
\text { Índice de vulnerabilidad hídrica, } \\
\text { condiciones año medio, } 2010\end{array}$ & SIAC (2016) \\
\hline Sensibilidad ambiental & Shape de áreas & Índice de sensibilidad ambiental & SIAC (2016) \\
\hline
\end{tabular}

Fuente: Elaboración propia.

Teniendo como base los factores geográficos y climáticos que inciden en la producción, se evaluó el comportamiento de las variables altitud, radiación solar, humedad relativa, precipitación y temperatura y se incluyó, además, la clasificación climática. También se consideraron 
factores limitantes, como aspectos de balance hídrico, suelos, indicadores hidrológicos de erosión y sensibilidad ambiental.

Para realizar la caracterización, se tomó la información secundaria disponible en el Sistema de Información Ambiental de Colombia (SIAC) y el Sistema de Información Geográfica para la Planeación y el Ordenamiento Territorial (SIGOT). La información obtenida para el Triángulo del Café fue espacializada empleando los sistemas de información geográfica y se utilizó el software ArcGIS 10.1, que permitió elaborar diferentes capas temáticas. Se tomó la información disponible en formato Shapefile y ráster y se realizó un cruce con la región de los ecotopos cafeteros.

\section{Resultados}

A continuación, se describen las principales características generales de los ecotopos cafeteros en el Triángulo del Café colombiano.

\subsection{Altitud}

El Triángulo del Café posee características únicas, dada su localización en las estribaciones de los ramales de la cordillera de los Andes; se caracteriza por ser una zona montañosa, con todos los pisos térmicos, y alberga una gran diversidad de espacios geográficos que proporcionan diversos ecosistemas, como bosques subandinos, andinos, alto andinos, páramos y nivales. La Figura 2 (a) presenta la variación altitudinal para el Triángulo del Café, que va desde los 123 m. s. n. m. hasta los 5.289 m. s. n. m., según el modelo de elevación digital del terreno de la NASA (2015).

En Caldas y Risaralda se evidencia la presencia de un relieve complejo. Las mayores altitudes se presentan en el Parque Nacional Natural los Nevados, en las cuencas de los ríos Chinchiná y Otún, respectivamente, y las menores altitudes están en los afluentes directos al Magdalena y el río San Juan Alto. En Quindío predominan las alturas entre 1.000 m. s. n. m. y 2.000 m. s. n. m. En la zona de los ecotopos cafeteros, las variaciones altitudinales van desde $456 \mathrm{~m}$. s. n. m. en Risaralda y 2.665 m. s. n. m. en el departamento de Caldas, en el municipio de Aguadas; sin embargo, la zona del cultivo del café típicamente varía entre 800 y 2.200 m. s. n. m.

\subsection{Biomas}

Según el mapa de biomas del SIAC, se presentan cuatro tipos en la zona de estudio: general (50 \%), orobiomas andinos del zonobioma de bosque húmedo tropical (34\%), orobiomas del zonobioma de bosque húmedo tropical $(15 \%)$ y zonobioma del bosque húmedo tropical (1\%). Para la región de los ecotopos cafeteros se tienen 15.957 ha en orobiomas andinos y 502.844 ha en ecosistemas transformados. 
Olga Lucía Ocampo López, Karen Castañeda Peláez, Jorge Julián Vélez Upegui
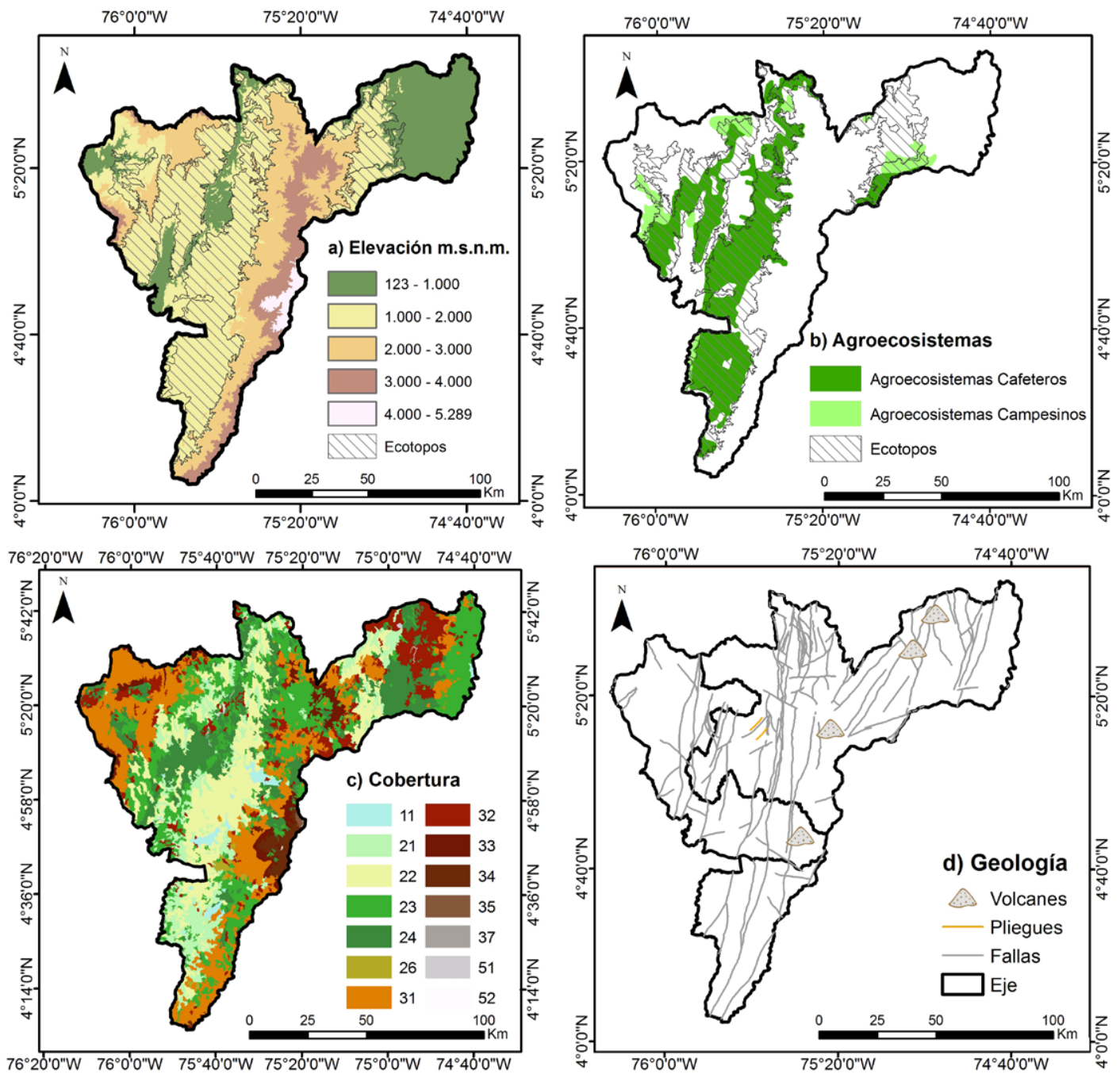

Figura 2. Mapas de elevación (a); agroecosistemas cafeteros y campesinos (b); coberturas (c) y localización de fallas, pliegues y volcanes $(d)$.

Fuente: Elaboración propia a partir de Cenicafé (2015), NASA (2015), SIAC (2016), Servicio Geológico Colombiano (2016) y SIGOT (2016).

\section{3 Áreas de reserva y protección}

El SIAC (2016) compila el Sistema Nacional de Áreas protegidas para Colombia, que comprende: los parques naturales nacionales, las reservas de la sociedad civil, las reservas forestales y los distritos de conservación de suelos, entre otros. Tomando como base esta información, se evidencia que la región de los ecotopos cafeteros no está bien delimitada, pues comprende áreas de protección, como el Parque Nacional Natural Selva de Florencia, el Parque Nacional Natural Tatamá y la
Reserva Forestal Central, protegida por Ley $2^{\text {a }}$; áreas de importancia para la conservación de aves (AICAs), como los bosques montanos del sur de Antioquia, así como los bosques del oriente de Risaralda, La Victoria y el cañón de los ríos Barbas y Bremen; distritos de conservación de suelos, como Barbas-Bremen, Campo Alegre, Guaca Rosario, Alto del Nudo y La Marcada; reservas naturales de la sociedad civil, como La Gaviota y Kasaguada; reservas forestales protectoras nacionales, como la quebrada La Nona Zurrumbo y Maní, la reserva forestal protectora regional que incluye Planalto y 
La Linda, y los parques naturales regionales Santa Emilia y Verdum.

\subsection{Agroecosistemas}

La Figura 2 (b) presenta la superficie cubierta por agroecosistemas cafeteros $(25,5 \%)$ y agroecosistemas campesinos mixtos $(4,8 \%)$. Para la región de los ecotopos, estos agroecosistemas representan el $56,3 \%$ y el $7,2 \%$ de la superficie, respectivamente. También se encuentran, en la franja apta para la caficultura, áreas rurales intervenidas (30,1\%), agroecosistemas empresariales de secano $(0,2 \%)$, bosques plantados $(2,3 \%)$ y áreas urbanas $(0,7 \%)$.

\subsection{Coberturas}

La distribución de la superficie por tipo de cobertura se resume en la Tabla 2 y se ilustra en la Figura 2(c). En el Triángulo del Café, la mayor proporción se encuentra en pastos, que equivalen a 281.637 ha, seguidos de los bosques naturales, con 247.285 ha, y los cultivos permanentes y semipermantes, con 211.900 ha. Para la región de los ecotopos cafeteros, los cultivos semipermanentes y permanentes ocupan la mayor extensión, con 193.561 ha.

\subsection{Geología}

El Triángulo del Café cuenta con una gran variedad de unidades geológicas de diferentes edades $\mathrm{y}$ orígenes que muestran el basamento de la cordillera Central y su historia volcánica reciente y subreciente. En la región de los ecotopos cafeteros se presentan diferentes fallas geológicas, como Samaná, Río Dulce, Santa Rosa, Palestina, Aranzazu, Honda, Apía, Quebrada Nueva, Armenia, Silvia-Pijao, Montenegro, Mistrató, Amurrapá, Cauca-Almaguer y San Jerónimo; en esta zona también se encuentra el volcán El Escondido, al oriente de Caldas.

\section{TABLA 2. USO Y COBERTURA DEL SUELO EN EL TRIÁNGULO DEL CAFÉ}

\begin{tabular}{|c|c|c|c|}
\hline \multirow{2}{*}{ Código } & \multirow{2}{*}{ Coberturas } & \multirow{2}{*}{$\begin{array}{c}\text { Triángulo del Café } \\
\text { Área \% }\end{array}$} & \multirow{2}{*}{$\begin{array}{c}\text { Ecotopos cafeteros } \\
\text { Área \% }\end{array}$} \\
\hline & & & \\
\hline 23 & Pastos & 21,8 & 15,0 \\
\hline 31 & Bosques naturales & 19,1 & 0,7 \\
\hline 22 & Cultivos semipermanentes y permanentes & 16,4 & 37,0 \\
\hline 24 & Áreas agrícolas heterogéneas & 15,2 & 15,0 \\
\hline 21 & Cultivos anuales o transitorios & 10,4 & 19,0 \\
\hline 32 & Vegetación secundaria & 9,3 & 4,0 \\
\hline 33 & Arbustales & 3,1 & 0,02 \\
\hline 34 & Herbazales & 1,8 & - \\
\hline 11 & Áreas urbanas & 1,3 & 2,0 \\
\hline 26 & Bosques plantados & 1,0 & 1,0 \\
\hline 51 & Aguas continentales naturales & 0,4 & - \\
\hline 35 & Zonas desnudas, sin o con poca vegetación & 0,1 & - \\
\hline 37 & Glaciares y nieves & 0,04 & - \\
\hline 52 & Aguas continentales artificiales & 0,01 & 0,02 \\
\hline
\end{tabular}

Fuente: Elaboración propia a partir de Cenicafé (2015) y SIGOT (2016). 


\subsection{Vocación de suelos}

El Triángulo del Café tiene una gran variabilidad de suelos por la diversidad de materiales de origen y la topografía. La vocación de los suelos para cultivos permanentes es presentada por la Unidad de Planificación Rural Agropecuaria (UPRA, 2016). Partiendo del mapa nacional, se digitalizó la información para el Triángulo del Café; el mapa se presenta en la Figura 3 (a). Las zonas aptas para cultivos permanentes se encuentran, en su mayoría, en la cuenca del río Cauca.
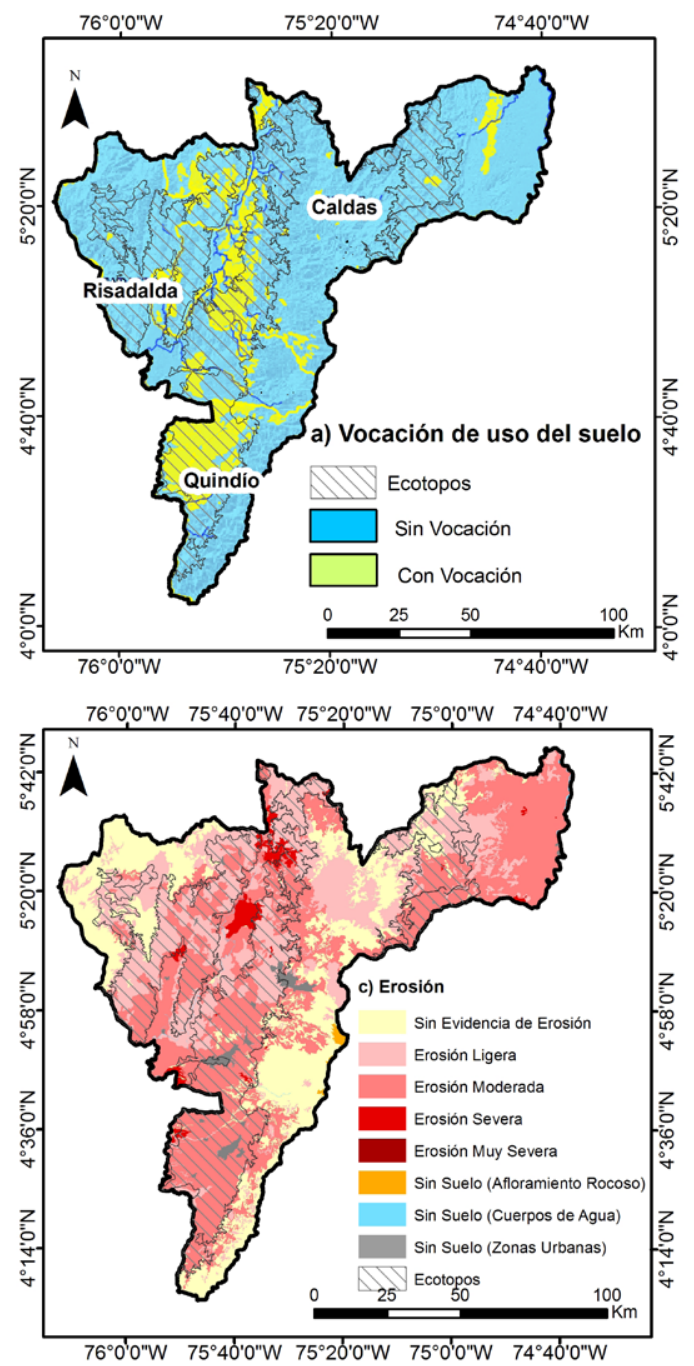

\subsection{Conflictos de uso del suelo}

El SIG-OT reporta la superficie del territorio nacional que se encuentra en conflicto de uso del suelo, y con esta información se elaboró el mapa de conflictos para el Triángulo del Café que se ilustra en la Figura 3 (b); los conflictos que se reportan son: sobreutilización (49\%), subutilización (20\%) y sin datos (31\%). Para la región de los ecotopos cafeteros, el porcentaje de superficie en conflicto es el siguiente: sobreutilización (46\%), subutilización (17\%) y sin datos (37\%).
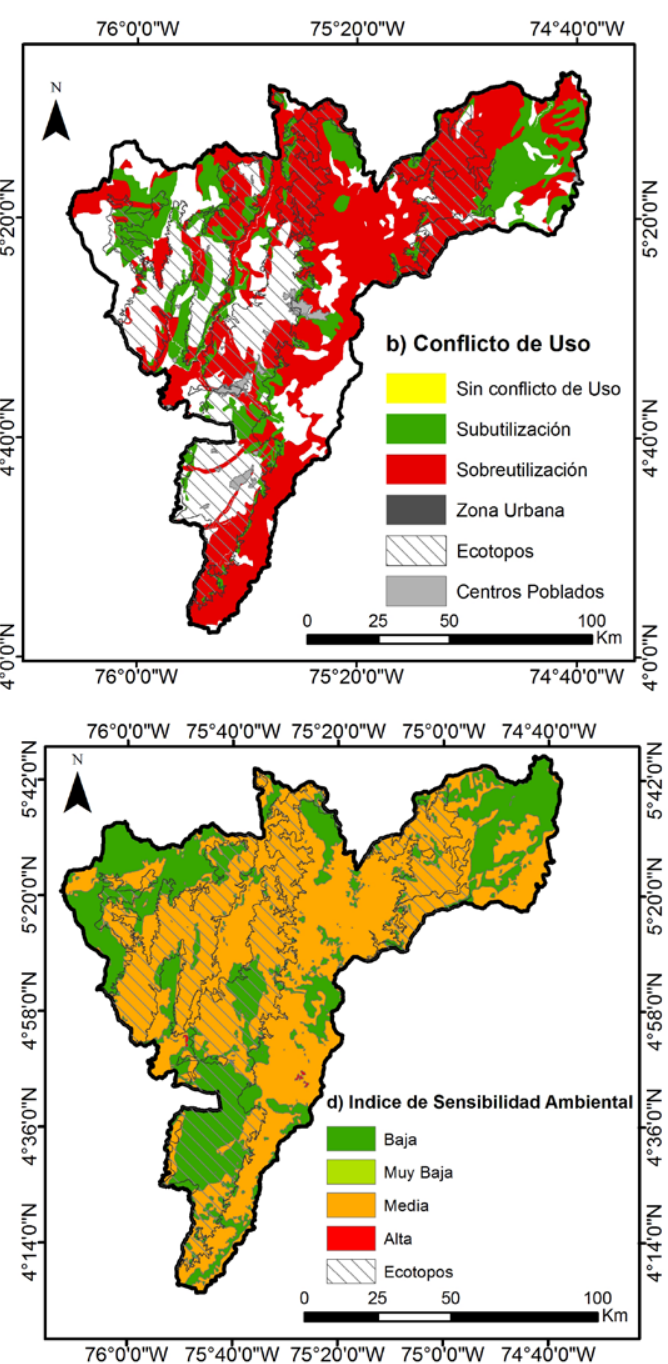

Figura 3. Mapas de vocación de uso del suelo (a); conflicto de uso (b); erosión (c) e índice de sensibilidad ambiental (d). Fuente: Elaboración propia a partir de (2015), SIAC (2016), SIG-OT (2016) y UPRA (2016). 


\subsection{Erosión}

Según el Instituto Nacional de Hidrología, Meteorología y Estudios Ambientales de Colombia (IDEAM, 2012), aproximadamente el $50 \%$ de las tierras continentales de Colombia presentan algún grado de degradación por erosión, el $5 \%$ por salinización y el $24 \%$ son susceptibles a la desertificación y otros procesos de degradación como la compactación, la disminución de la fertilidad, la sedimentación y la contaminación. El mapa de erosión del Triángulo del Café se ilustra en la Figura 3(c), según la información reportada por el SIAC (2016); la distribución porcentual por área es la siguiente: sin evidencia de erosión $23,7 \%$, erosión ligera $29,64 \%$, erosión moderada $43,3 \%$, erosión severa $2,2 \%$, erosión muy severa $0,003 \%$, sin suelo $1,1 \%$. Para la zona de los ecotopos cafeteros, la clasificación del grado de erosión es como sigue: sin evidencia 5,6 \%, ligera $37,5 \%$, moderada $54,4 \%$, severa $1,8 \%$, muy severa $0,004 \%$ y no suelo $0,7 \%$.

\subsection{Sensibilidad ambiental}

La Segunda Comunicación Nacional de Cambio Climático mide la sensibilidad ambiental en el territorio colombiano a escala municipal (IDEAM, 2010a). Para el Triángulo del Café, se muestra en la Figura 3(d). La distribución por área para las diferentes categorías del índice es la siguiente: muy baja $0,1 \%$, baja $34,2 \%$, media $65,6 \%$ y alta $0,1 \%$. Para la zona de los ecotopos cafeteros, la categorización es la siguiente: baja 29 \% y media $71 \%$.

\subsection{Radiación solar}

La radiación solar es necesaria para los procesos físicos y biológicos que ocurren en el cultivo del café y está determinada por el macroclima de la región, por las condiciones de nubosidad, las propiedades del follaje y la densidad de siembra, entre otras (Jaramillo, 2005). Por la posición geográfica, el país recibe abundante radiación solar; sin embargo, se presentan modificaciones en las cantidades diarias por los factores locales y la nubosidad; la variación interanual sigue un patrón bimodal (IDEAM, 2005; Ocampo, Vélez y Londoño, 2014; Ocampo y Vélez, 2015).

Según el mapa de radiación solar para el período 1980-2002 reportado por el SIAC (2016) (Figura 4[a]) para el Triángulo del Café, fluctúa entre 3,0 $\mathrm{kWh} / \mathrm{m}^{2}$ y $5,5 \mathrm{kWh} / \mathrm{m}^{2}$. En los ecotopos cafeteros se presenta variación entre $3,0 \mathrm{kWh} / \mathrm{m}^{2}$ hasta 5,0 $\mathrm{kWh} / \mathrm{m}^{2}$, con mayores valores en los municipios de Chinchiná, Palestina, Marsella, Belalcázar, La Virginia, San José, Risaralda, Belén de Umbría, Viterbo, Santuario, La Celia y Balboa. 

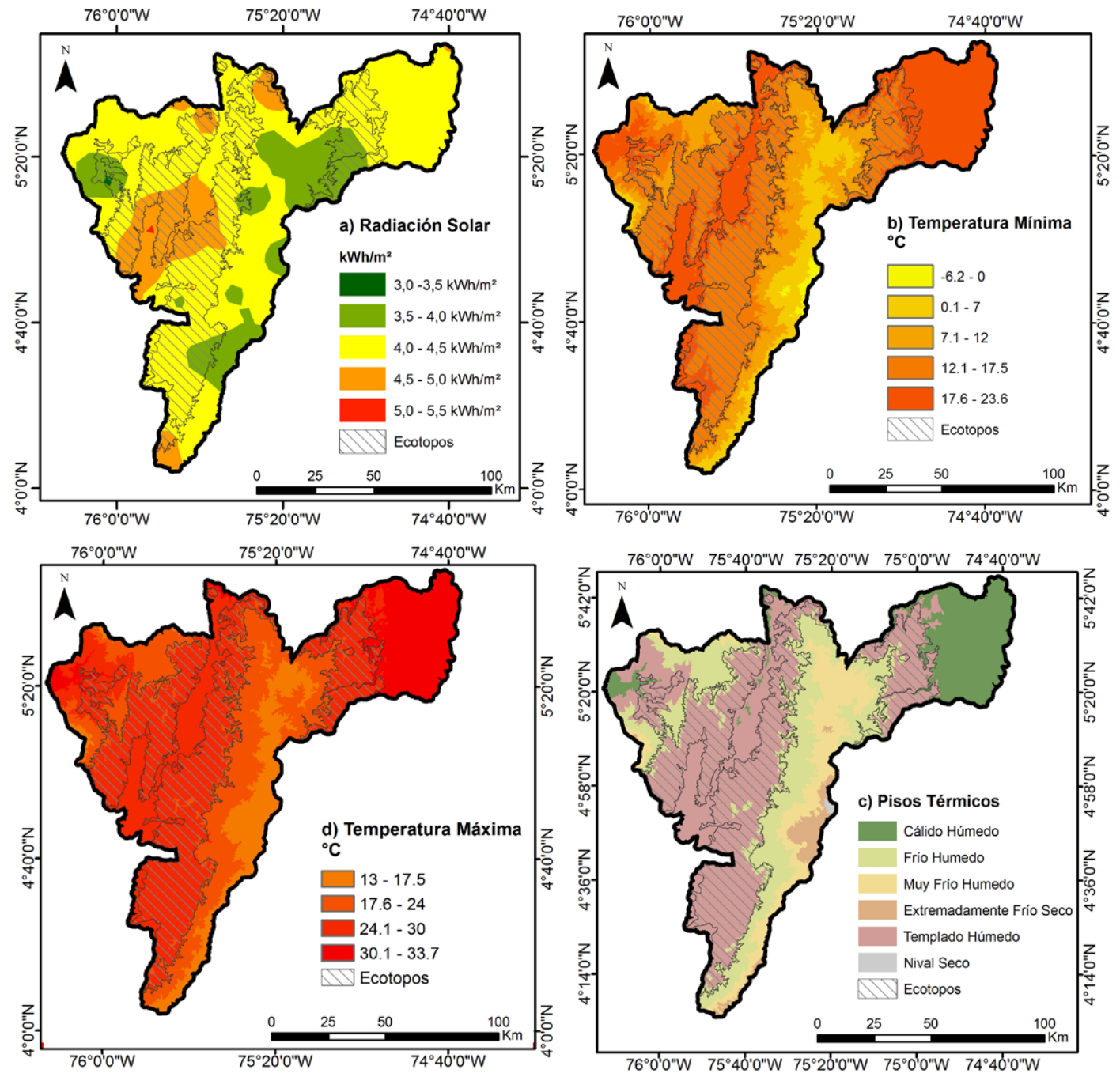

Figura 4. Mapas de radiación solar (a); temperatura mínima (b); temperatura máxima (c) y pisos térmicos (d). Fuente: Elaboración propia a partir de Cenicafé (2015), SIAC (2016) y WorldClim (2016).

\subsection{Temperaturas}

La temperatura es proporcional a la radiación incidente; sin embargo, existe desfase según las condiciones atmosféricas porque es afectada por los factores locales, la convección y la turbulencia del aire (IDEAM, 2005). La temperatura máxima se registra después del mediodía y la mínima un poco antes de la salida del sol. La tasa de variación de la temperatura con la altitud no es homogénea en el territorio colombiano, es del orden de $6,4^{\circ} \mathrm{C}$ por $\mathrm{km}$ en la zona andina colombiana (Ocampo y Vélez, 2015; Ocampo, Vélez y Londoño, 2014).
La información acerca de la variación espacial de la temperatura promedio del aire reportada por WorldClim (2015) para el período 1950-2000 en el Triángulo del Café es la siguiente: la temperatura mínima, que se ilustra en la Figura 4(b), oscila entre $-6,2{ }^{\circ} \mathrm{C}$ hasta $23,59^{\circ} \mathrm{C}$ con valores medios de $14,52{ }^{\circ} \mathrm{C}$ y una desviación estándar de $5,32{ }^{\circ} \mathrm{C}$. La temperatura media fluctúa entre $5,8{ }^{\circ} \mathrm{C}$ y $29,3{ }^{\circ} \mathrm{C}$ con valores promedio de $19,36^{\circ} \mathrm{C}$ y una desviación estándar de $5,87^{\circ} \mathrm{C}$. La temperatura máxima que se presenta en el mapa (Figura $4[\mathrm{c}]$ ) varía entre $13{ }^{\circ} \mathrm{C}$ a $33,7{ }^{\circ} \mathrm{C}$, con valores promedio de $24,86^{\circ} \mathrm{C}$ y una desviación estándar de $5,19{ }^{\circ} \mathrm{C}$. Para la región de 
los ecotopos cafeteros, los promedios de temperatura son los siguientes: mínima: $15,90{ }^{\circ} \mathrm{C}$; media: $20,17{ }^{\circ} \mathrm{C}$ y máxima: $26,2{ }^{\circ} \mathrm{C}$, mientras que las desviaciones estándar son $1,70,1,82$ y $1,64{ }^{\circ} \mathrm{C}$ respectivamente.

\subsection{Clasificación climática}

La clasificación climática está basada en patrones como la precipitación y la temperatura. Para la zona de estudio se evaluó la clasificación de Caldas-Lang (IDEAM, 2005), considerando la información reportada por el SIAC (2016) (Figura 4[c]) para el período 1971-2000. La región cafetera presenta una gran diversidad de climas por su gradiente altitudinal. Los ecotopos cafeteros se encuentran, en su mayoría, en el territorio templado-húmedo (800-1.800 m. s. n. m.), y la distribución por áreas es la siguiente: cálido-húmedo $1 \%$, frío-húmedo $15 \%$ y templado-húmedo $84 \%$.

\subsection{Cuencas hidrográficas}

El Triángulo del Café comprende doce cuencas hidrográficas, según los reportes del Estudio Nacional de Agua (IDEAM, 2014) registrados en los mapas del SIG-OT (2016), que son las siguientes: directos al Magdalena entre los ríos Guarinó, La Miel, río Tapias y otros directos al Cauca, río Pescador - RUT - Chanco - Catarina y Cañaveral, río Arma, río Chinchiná, río Frío y otros directos al Cauca, río Guarinó, río La Miel, río La Vieja, río Otún y otros directos al Cauca, río Risaralda, río San Juan.

\subsection{Precipitación}

En el Triángulo del Café, los regímenes de precipitación están determinados por el movimiento de la zona de confluencia intertropical (ZTIC) y factores físicos como la orografía (Ocampo y Vélez, 2015; Poveda, 2004). Con relación al ciclo anual, la precipitación en el Triángulo del Café exhibe un comportamiento bimodal con dos períodos más lluviosos en el año, entre abril-mayo y octubre-noviembre. El comportamiento de la precipitación promedio anual en el Triángulo del Café para el período 1971-2000 se muestra en la Figura 4(a); las mayores se dan en la cuenca del río La Miel-Samaná Sur y en la cuenca del río San Juan Alto, mientras que las menores se localizan en la zona del Parque Nacional Natural Los Nevados. En la zona de los ecotopos cafeteros, la precipitación oscila entre los 1.000 y los $7.000 \mathrm{~mm}$ para el período 1971-2000, con valores más altos en el municipio de Samaná, en Caldas, y en Pueblo Rico, en Risaralda, mientras que los menores valores se presentan en algunas zonas del noroccidente de Caldas, el suroccidente de Risaralda y el occidente del Quindío. El comportamiento de la precipitación total anual para el Triángulo del Café, según el Estudio Nacional de Agua (ENA) (IDEAM, 2014), para el período 1974-2010, presenta valores entre los 1.000 y los 7,000 mm; para el caso de la zona de cultivo de café, se encuentra en la franja entre 1.500 y $7.000 \mathrm{~mm}$, lo cual indica una inadecuada delimitación en el oriente de Caldas, pues se toman zonas con muy abundante precipitación.

Las anomalías en la precipitación por efecto de la variabilidad climática son presentadas por el ENA (2014) para el período 1974-2010. Durante el año húmedo (La Niña), las mayores anomalías se presentan en las cuencas de los afluentes directos al Magdalena y el río Risaralda, mientras que en años secos (El Niño) las mayores reducciones se presentan en los afluentes directos al Magdalena. Para la región de los ecotopos cafeteros, las anomalías en el año húmedo fluctúan entre 42,6 \% y 79,84 \%, con mayores valores en la cuenca del río Risaralda, mientras que en el año seco estas anomalías oscilan entre $-36,18 \%$ y $-53,25 \%$, con valores más extremos en la cuenca del río Tapias y otros directos al Cauca. 

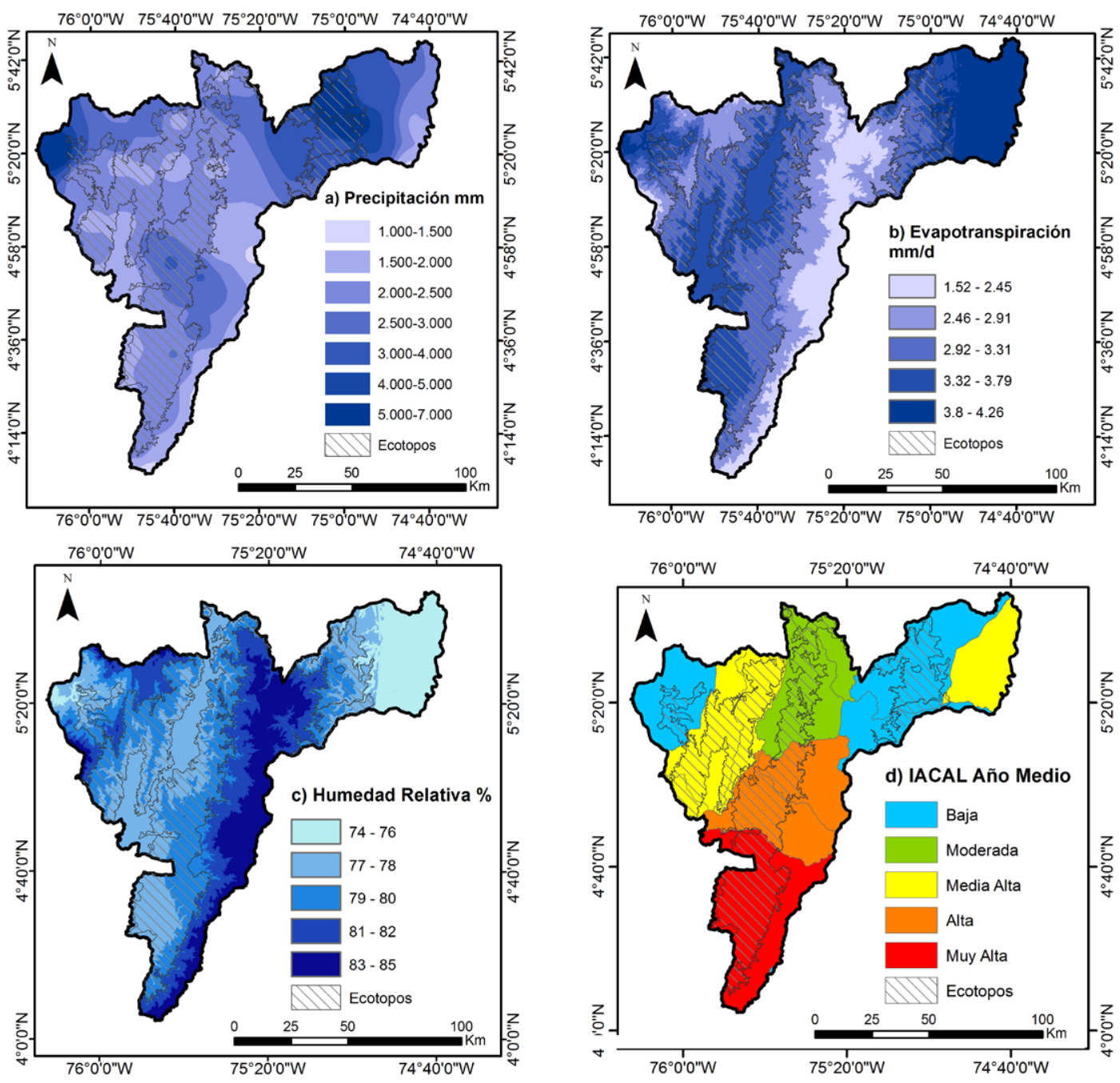

Figura 5. Mapas de precipitación (a); evapotranspiración (b); humedad relativa (c) y calidad de agua (d). Fuente: Elaboración propia a partir de SIAC (2016) y Cenicafé (2015).

\subsection{Balance hídrico}

El balance hídrico en una región lo componen las entradas y las salidas de agua; por lo tanto, debe considerar la lluvia, la evapotranspiración, el agua interceptada por la planta, la escorrentía y el agua almacenada en el suelo (Jaramillo, 2005). La evapotranspiración es el proceso por el cual el agua cambia de estado líquido a gaseoso directamente o a través de las plantas; puede estar representada por la evapotranspiración potencial (ETP), que contempla la pérdida de agua observada en una superficie por efecto de evaporación y transpira- ción y que ocurre con un adecuado abastecimiento de humedad al suelo (Ocampo, Vélez y Londoño, 2014). Para la estimación de la ETP en el Triángulo del Café que se muestra en la Figura 4(b), se tomó el modelo propuesto por Jaramillo (2005). Para las áreas de los ecotopos cafeteros, la ETP fluctúa entre 2,86 y $4,2 \mathrm{~mm} /$ día, con un promedio de 3,4 mm/día y una desviación estándar de 0,19 $\mathrm{mm} /$ día. La escorrentía comprende el exceso de precipitación que ocurre después de la lluvia y se mueve libremente sobre el terreno (Ocampo, Vélez y Londoño, 2014). La escorrentía promedio anual en el Triángulo del Café presenta variacio- 
nes desde los $800 \mathrm{~mm}$ hasta los $6.000 \mathrm{~mm}$. Los mayores valores se encuentran en la cuenca del río La Miel-Samaná Sur y la cuenca del río San Juan Alto. Para la región de los ecotopos cafeteros, fluctúa entre 1.000 y $5.000 \mathrm{~mm}$, según el mapa de escorrentía del SIAC (2016).

\subsection{Humedad relativa}

La humedad relativa es la relación porcentual entre la humedad de un espacio y la cantidad que ese volumen podría contener si estuviera saturado (Ocampo, Vélez y Londoño, 2014). En la zona andina colombiana, sigue un comportamiento bimodal estacional típico por efecto del movimiento de la ZCIT; por otra parte, es influenciada fuertemente por la altitud. Ocampo y Vélez (2015) plantean un modelo para su estimación durante el período 1981-2010 en el departamento de Caldas, que fue aplicado en este estudio; para el Triángulo del Café, varía entre el 74 y el $85 \%$, con un promedio de 78,86 \% y una desviación estándar de $2,56 \%$. Los menores valores se presentan en los valles de los ríos Magdalena y Atrato, mientras que más altos están en la cordillera Central. En los ecotopos cafeteros, la humedad relativa promedio anual multianual oscila entre el 75,3 y el $81,5 \%$, con valores medios de 78,2 \% y una desviación estándar de $0,81 \%$.

\subsection{Indicadores hidrológicos}

Los indicadores hidrológicos fueron obtenidos del Estudio Nacional de Agua (IDEAM, 2010), compilados por el SIAC (2016) para Colombia, y comprenden el índice de alteración potencial de la calidad (IACAL), el índice de uso de agua y el índice vulnerabilidad hídrica. El IACAL es un indicador de calidad del agua que tiene en cuenta la demanda biológica oxígeno (DBO), la diferencia entre la demanda química de oxígeno (DQO) y la DBO, los sólidos suspendidos totales (SST), el nitrógeno total (NT) y el fósforo total (PT) (IDEAM, 2010); entre más alto sea el índice, es mayor el grado de contaminación. Para el Eje Cafetero, en condiciones de año medio (Figura 4[d]), el IACAL es muy alto en la cuenca del río La Vieja; alto en las cuencas de los ríos Chinchiná y Otún y otros directos al Cauca, y medio alto en la cuenca del río Risaralda. En año seco, alcanza la categoría muy alto en las cuencas de los ríos La Vieja, Chinchiná, Otún y otros directos al Cauca.

El índice de uso de agua es la relación porcentual entre la demanda de agua y la oferta disponible (IDEAM, 2010). En condiciones de año medio, las cuencas de los ríos La Vieja, Otún y Chinchiná tienen un índice moderado, y en las demás no se tiene presión por el recurso. En condiciones de año seco, el índice es alto en las cuencas de los ríos Otún y La Vieja y moderado en Chinchiná y La Miel-Samaná Sur. El índice de vulnerabilidad hídrica mide la fragilidad del sistema hídrico para mantener una oferta para el abastecimiento de agua ante amenazas por períodos largos de estiaje o eventos como El Niño-Oscilación del Sur (ENOS, o ENSO en inglés) que podrían generar riesgos de abastecimiento; considera, por tanto, el índice de retención y regulación hídrica y el índice de uso del agua (IDEAM, 2010; Ocampo, Vélez y Londoño, 2014). En condiciones de año medio, la vulnerabilidad es media en las cuencas de los ríos Chinchiná, Otún y La Vieja; en las demás cuencas es baja. En año seco, el índice de vulnerabilidad hídrica es alto en las cuencas de los ríos Otún y La Vieja, y media en Chinchiná, Risaralda y La Miel-Samaná Sur.

\section{Conclusiones}

Los resultados de este estudio permiten una mejor comprensión de los factores climáticos y geográficos del Triángulo del Café y de la zona de los ecotopos cafeteros, que se compilan en la Tabla 3. Estos factores son determinantes en la productividad cafetera y, por tanto, brindan lineamientos para la ordenación y la planificación del territorio. La caracterización evidencia que es posible mejorar la delimitación de los ecotopos cafeteros, ya que es necesario excluir áreas naturales protegidas de orden nacional, departamental y municipal, así como reservas de la sociedad civil y reservas de la biosfera. Se requiere, además, tener en cuenta las condiciones climáticas apropiadas para el cul- 
tivo del café, como lluvias entre 1.800 y $2.800 \mathrm{~mm}$ mayor a seis horas de luz por día y humedad relapor año, temperaturas entre 18 a $23^{\circ} \mathrm{C}$, brillo solar tiva entre el 70 y el $85 \%$.

\section{TABLA 3. CARACTERIZACIÓN DE LOS ECOTOPOS CAFETEROS EN EL TRIÁNGULO DEL CAFÉ}

\begin{tabular}{|c|c|c|c|}
\hline Variables & Medida & Triángulo del Café & $\begin{array}{l}\text { Ecotopos } \\
\text { cafeteros }\end{array}$ \\
\hline $\begin{array}{l}\text { Altitud } \\
\text { m s. n. m. }\end{array}$ & Rango & $123-5289$ & $800-2200$ \\
\hline \multirow{4}{*}{$\begin{array}{l}\text { Biomas } \\
\% \text { área }\end{array}$} & General & $50 \%$ & \multirow{4}{*}{$\begin{array}{c}31 \% \\
66 \% \\
3 \%\end{array}$} \\
\hline & Orobiomas andinos & $34 \%$ & \\
\hline & Orobiomas del zonobioma de bosque húmedo tropical & $15 \%$ & \\
\hline & Zonobioma del boque húmedo tropical & $1 \%$ & \\
\hline \multirow{6}{*}{$\begin{array}{l}\text { Agroecosistemas } \\
\% \text { área }\end{array}$} & Agroecosistemas cafeteros & $25,5 \%$ & $56,3 \%$ \\
\hline & Agroecosistemas campesinos mixtos & $4,8 \%$ & $7,2 \%$ \\
\hline & Áreas rurales intervenidas no diferenciadas & $49,8 \%$ & $30,1 \%$ \\
\hline & Agroecosistemas empresariales & $0,6 \%$ & $0,2 \%$ \\
\hline & Bosques plantados & $3,4 \%$ & $2,3 \%$ \\
\hline & Otros & $15,9 \%$ & $3,9 \%$ \\
\hline \multirow{3}{*}{$\begin{array}{l}\text { Conflictos de uso de } \\
\text { suelo } \\
\% \text { área }\end{array}$} & Sobreutilización & $49 \%$ & $46 \%$ \\
\hline & Subutilización & $20 \%$ & $17 \%$ \\
\hline & Sin clasificación & $31 \%$ & $37 \%$ \\
\hline \multirow{6}{*}{$\begin{array}{l}\text { Erosión } \\
\% \text { área }\end{array}$} & Sin evidencia & $23,7 \%$ & $5,6 \%$ \\
\hline & Erosión ligera & $29,64 \%$ & $37,5 \%$ \\
\hline & Erosión moderada & $43,3 \%$ & $54,4 \%$ \\
\hline & Erosión severa & $2,2 \%$ & $1,8 \%$ \\
\hline & Erosión muy severa & $0,003 \%$ & $0,004 \%$ \\
\hline & Sin suelo & $1,1 \%$ & $0,7 \%$ \\
\hline \multirow{4}{*}{$\begin{array}{l}\text { Sensibilidad ambiental } \\
\% \text { área }\end{array}$} & Muy baja & $0,1 \%$ & \multirow{4}{*}{$\begin{array}{l}29 \% \\
71 \%\end{array}$} \\
\hline & Baja & $34,2 \%$ & \\
\hline & Media & $65,6 \%$ & \\
\hline & Alta & $0,1 \%$ & \\
\hline $\begin{array}{l}\text { Radiación solar } \\
\mathrm{kWh} / \mathrm{m}^{2}\end{array}$ & Promedio & $3,0-5,5$ & $3,0-5,0$ \\
\hline \multirow{3}{*}{$\begin{array}{l}\text { Temperaturas } \\
\text { promedio } \\
{ }^{\circ} \mathrm{C}\end{array}$} & Mínimas & $-6,2-23,59$ & $9-21,6$ \\
\hline & Medias & $5,8-29,3$ & $14,3-25,3$ \\
\hline & Máximas & $13,0-33,7$ & $19,6-31,8$ \\
\hline $\begin{array}{l}\text { Precipitación } \\
\mathrm{mm}\end{array}$ & Promedio & $1000-7000$ & $1500-7000$ \\
\hline \multirow{2}{*}{$\begin{array}{l}\text { Anomalías precipitación } \\
\%\end{array}$} & Año seco (El Niño) & $-58 ;-34,6$ & $-53,25 ;-36,2$ \\
\hline & Año húmedo (La Niña) & $39,9-87,1$ & $42,6-79,8$ \\
\hline $\begin{array}{l}\text { Evapotranspiración } \\
\mathrm{mm} / \mathrm{d}\end{array}$ & Promedio & $1,6-4,5$ & $2,86-4,2$ \\
\hline $\begin{array}{l}\text { Escorrentía } \\
\mathrm{mm}\end{array}$ & Promedio & $800-6.000$ & $1.000-5.000$ \\
\hline $\begin{array}{l}\text { Humedad relativa } \\
\%\end{array}$ & Promedio & $74-85$ & $75,3-81,5$ \\
\hline
\end{tabular}

Fuente: Elaboración propia. 
Para el manejo y protección del Paisaje Cultural Cafetero es fundamental la sostenibilidad ambiental (Ministerio de Cultura, 2011); en este sentido, es necesario reconocer la sensibilidad ambiental media de los ecotopos cafeteros del Triángulo del Café. Se requiere establecer planes de manejo para las zonas con conflicto de uso del suelo por sobreutilización, que ascienden al $17 \%$; planes de control de erosión en más de la mitad del territorio que presenta índices de erosión moderada $(54,4 \%)$ y severa $(1,8 \%)$ y dar prioridad al cumplimiento de los planes de gestión integral del recurso hídrico en las cuencas de los ríos La Vieja, Chinchiná, Otún y otros directos al Cauca que presentan presión por el recurso hídrico, no solo por sus índices de uso de agua, sino también por la vulnerabilidad hídrica y los altos niveles de contaminación.

La información disponible en el Sistema de Información Ambiental de Colombia permite caracterizar en un territorio los agroecosistemas, los biomas, las áreas de reserva y protección, la zonificación de la degradación de suelos por erosión, la radiación solar global, la precipitación total anual y diferentes indicadores hidrológicos. Por otra parte, esta información facilita la comprensión del cambio en variables e indicadores hidrológicos por la variabilidad climática, específicamente en años húmedos por efecto del fenómeno de La Niña y años secos con ocurrencia del fenómeno de El Niño. La metodología empleada en este estudio puede aplicarse en diferentes temáticas del ordenamiento territorial, como los planes de ordenamiento de cuencas hidrográficas. El uso de SIG permite generar una cartografía de los ecotopos cafeteros en el Triángulo del café que posibilita su integración en estudios regionales y nacionales y que puede ser actualizada y complementada con nueva información, tal como lo plantea Salinas Chaves (2013).

La ley sobre la transparencia y el derecho a la información pública nacional (Ley 1712 de 2014) ha llevado a un cambio estructural en el acceso a la información geográfica en Colombia, lo que permite avanzar en la comprensión del territorio, conocer sus potenciales y limitaciones y sus posibles aplicaciones, como se presenta en este estudio de caracterización de los ecotopos cafeteros en el Triángulo del café. 


\section{Referencias}

Arcila, J., Farfán, F., Moreno, A., Salazar, F. e Hincapié, E. (2007). Sistema de producción de café en Colombia. Chinchiná, Colombia: Cenicafé.

Calambas, R. (2009). Estudio de las propiedades físicas y químicas del suelo, en sistemas de producción de café orgánico y tradicional en los municipios de Caldono, Morales y Piendamó en el departamento del Cauca. Palmira, Colombia: Universidad Nacional de Colombia, sede Palmira.

Camayo-Vélez, G. C., Chávez-Córdoba, B., Arcila-Pulgarín, J., Jaramillo-Robledo, A. (2003). Desarrollo floral del cafeto y su relación con las condiciones climáticas de Chinchiná-Caldas. Cenicafé, 54(1), 35-49.

Carr, M. K. V. (2001). The water relations and irrigation requirements of coffee. Experimental Agriculture, 37(1), 1-36.

Centro Nacional de Investigaciones del Café (Cenicafé) (2015). Ecotopos cafeteros Colombia shape. Chinchiná, Colombia: Cenicafé.

Crisosto, D., Grantz, F. C. y Meinzer, E. C. (1992). Effects of the water deficit on flower open in coffee (Coffea arabica L.). Tree Physiology, 10(2), 127-139.

Federación Nacional de Cafeteros de Colombia (2015). Comportamiento de la Industria Cafetera Colombiana 2014. Bogotá, Colombia: Federación Nacional de Cafeteros.

Gliessman, S. R. (2002). Agro ecología. Procesos ecológicos en agricultura sostenible. San José, Costa Rica: CATIE.

Gómez, L., Caballero, A. y Baldión, J. (1991). Ecotopos cafeteros de Colombia. Bogotá, Colombia: Federación Nacional de Cafeteros de Colombia.

Havlin, J. L., Beaton, J. D., Tisdale, S. L. y Nelson, W. L. (1999). Soil fertility and fertilizers: an introduction to nutrient management. $6^{a}$ Ed. Upper Saddle River, New Jersey: Prentice Hall.

Instituto Nacional de Hidrología, Meteorología y Estudios Ambientales de Colombia (IDEAM) (2010a). Segunda Comunicación Nacional ante la Convención Marco de las Naciones Unidas sobre Cambio Climático. Bogotá, Colombia: IDEAM, Ministerio Ambiente y Desarrollo Sostenible.

Instituto Nacional de Hidrología, Meteorología y Estudios Ambientales de Colombia (IDEAM) (2010). Estudio Nacional del Agua 2010. Bogotá, Colombia: IDEAM, Ministerio Ambiente y Desarrollo Sostenible.

Instituto Nacional de Hidrología, Meteorología y Estudios Ambientales de Colombia (IDEAM) (2012). Programa nacional de monitoreo y seguimiento de la degradación de suelos y tierras en Colombia. Bogotá, Colombia: IDEAM, Ministerio Ambiente y Desarrollo Sostenible.

Instituto Nacional de Hidrología, Meteorología y Estudios Ambientales de Colombia (IDEAM) (2014). Estudio Nacional del Agua 2014. Bogotá, Colombia: IDEAM, Ministerio Ambiente y Desarrollo Sostenible. 
Instituto de Hidrología, Meteorología y de Estudios Ambientales (IDEAM) (2005). Atlas climatológico de Colombia. Bogotá, Colombia: IDEAM, Ministerio de Ambiente, Vivienda y Desarrollo Territorial.

Jaramillo, A. (2005). Clima andino y café en Colombia. Chinchiná, Colombia: Cenicafé.

Ley 1712 de 2014 (6 de marzo), Por medio de la cual se crea la Ley de Transparencia y del Derecho de Acceso a la Información Pública Nacional y se dictan otras disposiciones. Diario Oficial n. ${ }^{\circ}$ 49.084. Recuperado de http://www. secretariasenado.gov.co/senado/basedoc/ley_1712_2014.html.

Lin, B. (2007). Agroforestry management as an adaptive strategy against potential microclimate extremes in coffee agriculture. Agricultural and Forest Meteorology, 144, 875-94.

Ministerio de Cultura (2011). Paisaje Cultural Cafetero. Un paisaje cultural productivo en permanente desarrollo. Bogotá, Colombia: Ministerio de Cultura de Colombia, Dirección de Patrimonio.

National Aeronautics and Space Administration (NASA) (2015). Shuttle Radar Topograpy Mission. Recuperado de: http://www2.jpl.nasa.gov/srtm/.

Ocampo, O. (2017). Modelación hidrológica y agronómica de los efectos del cambio y la variabilidad climática en la producción cafetera de Caldas. Manizales, Colombia: Universidad Nacional de Colombia, sede Manizales.

Ocampo, O., Vélez, J. J. y Londoño, A. (2014). Análisis de vulnerabilidad hídrica de la cuenca del río Chinchiná. Manizales, Colombia: Universidad Nacional de Colombia, sede Manizales.

Ocampo, O. y Vélez, J. J. (2015). Análisis climatológico para el departamento de Caldas. En: Entendimiento de fenómenos ambientales mediante análisis de datos (pp. 1-37). Manizales, Colombia: Universidad Nacional de Colombia, sede Manizales.

Paes, A. (1985). Florescimiento e fructificacao de café arabica nas diferentes regioes (cafeeiras) do Brasil. Pesquisa Agropecuaria Brasileira, 20(7), 831-839.

Poveda, G. (2004). La hidroclimatología de Colombia. Una síntesis desde la década inter-decadal hasta la escala diurna. Revista de la Academia Colombiana de Ciencias, 28(107), 201-222.

Poveda, G., Turbay, S., Vélez, J. J., Ocampo, O. L., Acevedo, E. y Bedoya, M. (2014). iNo sé qué vamos a hacer con estos climas! Vulnerabilidad y adaptación a las variaciones climáticas extremas en la cuenca de la quebrada Los Cuervos, afluente del río Chinchiná, Colombia. Medellín, Colombia: Universidad Nacional de Colombia, Universidad de Antioquia, IDRC-CIHR-NSER.

Ramírez, V. H., Arcila, J., Jaramillo, A., Rendón, J. R., Cuesta, G., García, J. C., Menza, H. D., Mejía, C. G., Montoya, D. F., Mejía, J. W., Torres, J. C., Sánchez, P. M., Baute, J. E. y Peña, J. (Mayo, 2011). Variabilidad climática y la floración del café en Colombia. Avances Técnicos Cenicafé, 407, 1-8.

Salinas Chaves, E. (2013). Reflexiones acerca del papel del ordenamiento territorial en la planificación y gestión ambiental. Perspectiva Geográfica, 18(1), 141-156. 
Servicio Geológico Colombiano (2016). Mapa geológico de Colombia 2015. Recuperado de http://www2.sgc.gov.co/Geologia/Mapa-geologico-de-Colombia.aspx.

Sistema de Información Ambiental de Colombia (SIAC) (2016). Catálogo de mapas. Recuperado de: http://www.siac.gov.co/Catalogo_mapas.html.

Sistema de Información Geográfica para la Planeación y el Ordenamiento Territorial (SIG-OT) (2016). Mapas temáticos predefinidos - Nacional. Recuperado de: http:// sigotn.igac.gov.co/sigotn/frames_pagina.aspx.

Turbay, S., Nantes, B., Jaramillo, F., Vélez, J. J. y Ocampo, O. L. (2014). Adaptación a la variabilidad climática entre los caficultores de las cuencas de los ríos Porce y Chinchiná, Colombia. Investigaciones Geográficas, Boletín del Instituto de Geografía, 85, 95-112. https://doi.org/10.14350/rig.42298.

Unidad de Planificación Rural Agropecuaria (UPRA) (2016). Mapa de vocación de uso de suelo. Recuperado de: http://www.upra.gov.co/.

WorldClim - Global Climate Data (2016). Free climate data for ecological modeling and GIS. Recuperado de: http://www.worldclim.org/.

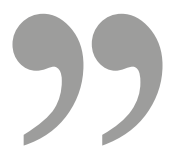

